\title{
Evaluation of the benefit of post-mastectomy radiotherapy in patients with early-stage breast cancer: A propensity score matching study
}

\author{
WENJIE SHI $^{1 *}$, YOUHONG LUO ${ }^{*}$, DONGKANG ZHAO ${ }^{1}$, HAO HUANG $^{1}$ and WEIYI PANG ${ }^{2}$ \\ ${ }^{1}$ Department of Breast Surgery, Affiliated Hospital of Guilin Medical University; ${ }^{2}$ Department of Environmental Health and \\ Occupational Medicine, School of Public Health, Guilin Medical University, Guilin, Guangxi 541000, P.R. China
}

Received March 8, 2018; Accepted February 14, 2019

DOI: $10.3892 / \mathrm{ol} .2019 .10197$

\begin{abstract}
The present study aimed to evaluate the significance of post-mastectomy radiotherapy (PMRT) in patients with early stage (T1-2) breast cancer. The Surveillance, Epidemiology, and End Results database was searched, and data on female patients with early stage (T1-2) breast cancer with 1-3 positive axillary lymph nodes (LNs) were extracted. Patients were subdivided into two groups: Those who had received PMRT and those who had not (no PMRT). Data from the two groups were analyzed in order to identify associations between PMRT status, breast cancer-specific survival (BCSS) probability and overall survival (OS) probability using multivariate Cox proportional hazards regression and propensity score matching models. A total of 7,316 patients were included in the analysis. Prior to propensity score matching, outcome probabilities were increased in the PMRT group, compared with the no PMRT group (BCSS probabilities: 92.0 vs. $90.1 \%$, respectively, $\mathrm{P}=0.015$; OS probabilities: 89.8 vs. $86.0 \%$, respectively, $\mathrm{P}<0.001)$. In multivariate analyses, tumor location was not identified as being a risk factor for BCSS (hazard ratio, 0.917; 95\% confidence interval, 0.772-1.090; $\mathrm{P}=0.326$ ). Following propensity score matching, differences between the two treatment groups (PMRT and no PMRT) in terms of their BCSS scores remained significant (93.7 vs. 90.1\%, respectively; $\mathrm{P}=0.007)$. Compared with the no PMRT group, the OS probabilities of the PMRT group were increased (89.4 vs. $86.0 \%$; $\mathrm{P}=0.025)$. In conclusion, the present results indicated that
\end{abstract}

Correspondence to: Dr Weiyi Pang, Department of Environmental Health and Occupational Medicine, School of Public Health, Guilin Medical University, 1 Zhiyuan Road, Guilin, Guangxi 541000, P.R. China

E-mail: p.weiyi@live.cn

*Contributed equally

Key words: breast cancer, post-mastectomy radiotherapy, propensity score matching
PMRT may benefit the prognosis of patients with breast cancer with early stage disease (T1-2), and those with one to three positive axillary LNs.

\section{Introduction}

Breast cancer is currently the most common cancer type in females globally, and its incidence has increased significantly in 2017, with a growing trend towards occurrence at a younger age $(1,2)$. At present, mastectomy (MAS) is generally considered to be the current method of choice for treatment of early-stage breast cancer $(3,4)$. However, Veronesi et al (5) reported that breast conserving surgery (BCS) and MAS are appropriate surgical options for the treatment of patients with early-stage breast cancer, and that there is no significant difference between the two procedures regarding the rate of local recurrence and overall survival (OS). Additionally, it has been observed that patients who underwent MAS exhibited aggressive clinicopathological factors, including young age of onset, large tumor size, high tumor grade, lymphovascular invasion or positive/close resection margin $(6,7)$. Postoperative radiotherapy has been reported as necessary in these patients in order to achieve a positive prognosis $(8,9)$. Other research demonstrated that post-MAS radiotherapy (PMRT) has a strong potential to produce a positive prognosis in females with stage T3-T4 breast cancer, or those where the cancer has spread to four or more positive axillary lymph nodes (LNs) $(10,11)$. Furthermore, a large retrospective study indicated that patients with node-negative breast cancer may also benefit from PMRT (12). However, the use of PMRT to improve breast cancer-specific survival (BCSS) and OS rates in early-stage (T1-2) breast cancer, and those with one to three positive axillary LNs, is controversial (13), due to limited clarity in the current evidence and a lack of data from large, high-quality clinical studies.

In observational studies, treatment options are frequently influenced by an individual's characteristics $(14,15)$. Furthermore the baseline characteristics of individuals who receive treatment are frequently systematically different from those of untreated individuals (16). Therefore, it is important to take into account the baseline characteristics of the treatment group and the group who did not receive treatment when assessing the efficacy of a 
treatment (17). Due to this, researchers are increasingly adopting the propensity score matching method in such studies in order to reduce the influence of confounding variables when using observational data (18).

Despite the controversy regarding its use in the treatment of early-stage breast cancer, postoperative radiotherapy has gained acceptance as an integral part of early-stage breast cancer treatment. The purpose of the present study was to investigate the BCSS and OS rates of a PMRT group and a group that did not receive PMRT after receiving MAS using propensity score matching.

\section{Patients and methods}

Participants. Female patients with breast cancer who had been diagnosed between January 2004 and November 2014 were selected from the Surveillance, Epidemiology and End Results cancer statistics database (https://seer.cancer.gov/; November 2016 edition), which included data from 18 population-based cancer registries in USA. The minimum age of the selected patients was 21 years and the maximum age was 94 years. Participant data was considered eligible on the precondition that the subject had received MAS only. Furthermore, only patients with unilateral lesions, pathologically confirmed infiltrating duct carcinoma (international classification of disease-O-3; 8500/3) and those who had early stage (T1-2) breast cancer with one to three positive axillary LNs were included. Patients were excluded if they had an uncertain American Joint Committee on Cancer (AJCC) stage of T or $\mathrm{N}$, or if they had other accompanying malignant tumors (19). Furthermore, individuals who had developed other malignant tumors prior to or during their breast cancer were excluded. Additionally, individuals with an unknown survival month and/or cause of mortality were also excluded from the present study. Individuals whose tumor grade at diagnosis was ambiguous were also excluded from the analysis.

Based on the aforementioned inclusion and exclusion criteria, data associated with the following variables were extracted from the database: Patient ID, race, age at diagnosis, marital status at diagnosis, tumor size, tumor grade, tumor laterality (left or right breast), number of positive nodes, primary site, AJCC 6th Tstage, surgery of the primary site, radiation treatment sequence and chemotherapy.

Study endpoint and statistical analyses. There were endpoints included in the analysis. BCSS was defined as the duration from the date of diagnosis to the date of last follow-up or mortality from breast cancer and OS was defined as the duration from the date of diagnosis to the date of last follow-up or mortality from any cause. Unpaired Student's t-test was used to identify differences between groups where data (variables) were continuous. A $\chi^{2}$ test was used to identify differences between groups involving categorical variables. Furthermore, propensity score matching was used to estimate the effects of treatment (PMRT) on the endpoint measures with reduced selection bias.

Prior to data analysis, a logistic regression model of the entire dataset was constructed in order to identify the main factors impacting outcomes in the treatment (PMRT) and control groups (no PMRT). The following variables were then filtered out of the model: Patient age, number of positive nodes, tumor grade and chemotherapy. Subsequently, the regression model was applied to the software package MatchIT in R version 3.4 .2 (https://www.r-project.org/) to calculate propensity scores representing the estimated probability of a patient in the group that received no PMRT based upon each participant's baseline characteristics. In applying this approach, there is evidence that the statistical efficiency does not significantly increase but the workload increases when the match ratio exceeds 1:4 $(20,21)$. Therefore, under the premise of ensuring maximum utilization of the data, patients without PMRT treatment were matched to patients with PMRT treatment with the closest propensity score based on a control ratio of 1:4 using a nearest neighbor algorithm approach with no replacement. Matching was restricted to being within the region of common support. A variety of checks were performed to ensure the adequacy of the model. The balance of means and variances of converts after matching was checked by examining the standardized mean differences between patients with no PMRT and PMRT prior to and following matching. Notably, following matching, the Student's t-test should be no longer significant. Furthermore, the histogram and kernel density of the probability distribution of propensity scores were examined prior to and following matching.

The Kaplan-Meier method was used to estimate the likelihood of BCSS and OS following propensity score matching. To compare survival between groups, the log-rank test was used. The hazard ratios (HR) and associated $95 \%$ confidence intervals (CIs) for BCSS and OS were examined using the Cox proportional hazards regression model. Variables exhibiting significant differences between groups upon univariate analysis were included in the multivariate analysis. All data were processed using the R soft version 3.4.2 and SPSS version 20.0 (IBM Corporation, Armonk, NY, USA). $\mathrm{P}<0.05$ was considered to indicate a statistically significant difference and all statistical tests were conducted as two-tailed tests.

\section{Results}

Characteristics of the patient groups. A total of 7,316 female patients with breast cancer who had been diagnosed between January 2004 and November 2014 were included in the analysis. Patients were divided into two groups based on whether or not they had received PMRT. The baseline characteristics of the patients are presented in Table I. The mean age and proportion of patients with T1 stage cancer were significantly increased among those who did not receive PMRT, compared with those who did receive PMRT $(\mathrm{P}<0.001)$. Patients who had received radiation therapy exhibited more advanced stage tumors (stage III and IV) and had an increased probability of refusing chemotherapy, compared with those who did not receive PMRT. Significant differences between the two treatment groups (no PMRT and PMRT groups) were also observed in terms of race, year of diagnosis and number of positive nodes (Table I). Following propensity score matching, the significant differences between the two treatment groups that were observed at baseline were no longer apparent (Table I). 
Table I. Baseline clinical characteristics of study participants who received or did not receive PMRT prior to and following propensity score matching.

\begin{tabular}{|c|c|c|c|c|c|c|}
\hline \multirow[b]{2}{*}{ Characteristic } & \multicolumn{3}{|c|}{ Prior to matching } & \multicolumn{3}{|c|}{ Following matching } \\
\hline & PMRT $(n=6931)$ & No PMRT (n=385) & P-value & $\operatorname{PMRT}(n=1540)$ & No PMRT $(\mathrm{n}=385)$ & P-value \\
\hline Age, mean \pm SD & $52.4 \pm 12.8$ & $60.6 \pm 14.1$ & $<0.001$ & $70.0 \pm 14.0$ & $60.6 \pm 14.1$ & 0.676 \\
\hline Race $(\%)$ & & & 0.030 & & & 0.997 \\
\hline White & $5,250(75.7)$ & $304(79.0)$ & & $1,215(78.9)$ & $304(79.0)$ & \\
\hline Black & $891(12.9)$ & $32(8.3)$ & & $127(8.2)$ & $32(8.3)$ & \\
\hline Other & $790(11.4)$ & $49(12.7)$ & & $198(12.9)$ & $49(12.7)$ & \\
\hline Location of tumor $(\%)$ & & & 0.214 & & & 0.915 \\
\hline Nipple/central & $545(7.9)$ & $43(11.2)$ & & $150(9.7)$ & $43(11.2)$ & \\
\hline Upper-inner & $728(10.5)$ & $37(9.6)$ & & $160(10.4)$ & $37(9.6)$ & \\
\hline Lower-inner & $414(6.0)$ & $22(5.7)$ & & $87(5.6)$ & $22(5.7)$ & \\
\hline Upper-outer & $2,804(40.5)$ & $141(36.6)$ & & $593(38.5)$ & $141(36.6)$ & \\
\hline Lower-outer & $664(9.6)$ & $41(10.6)$ & & $147(9.5)$ & $41(10.6)$ & \\
\hline Overlapping lesion & $1,776(25.6)$ & $101(26.2)$ & & $403(26.2)$ & $101(26.2)$ & \\
\hline T stage $^{\mathrm{a}}(\%)$ & & & 0.028 & & & 0.953 \\
\hline $\mathrm{T} 1$ & $2,292(33.1)$ & $149(38.7)$ & & $601(39)$ & $149(38.7)$ & \\
\hline $\mathrm{T} 2$ & 4,637 (66.9) & $236(61.3)$ & & $939(61)$ & $236(61.3)$ & \\
\hline Node, mean \pm SD & $1.77 \pm 0.80$ & $1.55 \pm 0.72$ & $<0.001$ & $1.57 \pm 0.73$ & $1.55 \pm 0.72$ & 0.639 \\
\hline Tumor size (\%) & & & 0.735 & & & 0.645 \\
\hline$<2 \mathrm{~mm}$ & $25(0.4)$ & $1(0.3)$ & & $2(0.1)$ & $1(0.3)$ & \\
\hline $2-5 \mathrm{~mm}$ & $80(1.2)$ & $6(1.6)$ & & $17(1.1)$ & $6(1.6)$ & \\
\hline$>5 \mathrm{~mm}$ & $6,826(98.5)$ & $378(98.2)$ & & $1,521(98.8)$ & $378(98.2)$ & \\
\hline Tumor grade (\%) & & & $<0.001$ & & & 0.786 \\
\hline $\mathrm{I}$ & $507(7.3)$ & $53(13.8)$ & & $209(13.6)$ & $53(13.8)$ & \\
\hline II & $2,685(38.7)$ & $182(47.3)$ & & $771(50.1)$ & $182(47.3)$ & \\
\hline III & $3,693(53.3)$ & $149(38.7)$ & & $556(36.1)$ & $149(38.7)$ & \\
\hline IV & $46(0.7)$ & $1(0.3)$ & & $4(0.3)$ & $1(0.3)$ & \\
\hline Chemotherapy (\%) & & & $<0.001$ & & & 0.918 \\
\hline Yes & $6,112(88.2)$ & $188(48.8)$ & & $759(49.3)$ & $188(48.8)$ & \\
\hline No/unknown & $819(11.8)$ & $197(51.2)$ & & $781(50.7)$ & $197(51.2)$ & \\
\hline
\end{tabular}

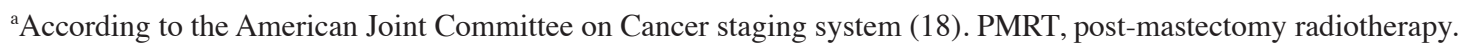

The univariate analysis predicted the major risk factors for BCSS among the PMRT and no PMRT groups to include age, race, number of positive nodes and $\mathrm{T}$ stage. Tumor location was not predicted to be a significant risk factor for BCSS (HR, 0.917; 95\% CI, 0.772-1.090; $\mathrm{P}=0.326$ ). The multivariate analysis predicted the significant risk factors for BCSS to be age (HR, 1.010; 95\% CI, 1.004-1.017; $\mathrm{P}=0.001)$ and $\mathrm{T}$ stage (HR, 0.395; 95\% CI, 0.319-0.489; P<0.001).

Oncological outcomes. When the unmatched patient data were analyzed, the median follow-up duration was determined to be significantly increased in the PMRT group, compared with the group that received no PMRT (113.89 vs. 103.17 months; $\mathrm{P}<0.001$ ). Additionally, the BCSS and OS probabilities of the PMRT group were increased, compared with those of the no PMRT group (BCSS probabilities: 92.0 and $90.13 \%$, respectively, $\mathrm{P}=0.015$; OS probabilities: 89.8 and 86.0 , respectively, $\mathrm{P}<0.001$; Fig. 1).
Following nearest-neighbor matching using the propensity scores, the histograms prior to and following matching were determined (Fig. 2). The histograms prior to matching on the left differed in raw treated and control graphs. However, the right histograms in matched treated and control data were similar. In summary, the numerical and visual data indicated that the matching was successful. The distributions of propensity scores prior to and following matching were indicated (Fig. 3). The jitter plot demonstrated that the baseline differences between the treatment (PMRT) and control (no PMRT) groups were marginal. A total of 1,540 cases in the PMRT group were matched to the group that did not receive PMRT, and 5,391 cases in the PMRT were discarded. Following matching, the analysis revealed that the BCSS probabilities following no PMRT were significantly reduced, compared with those of the PMRT group (90.1 vs. $93.7 \%$; $\mathrm{P}=0.007)$. Notably, the incidence of cumulative hazard events was $163 / 1,540$ (10.6\%) in the PMRT group and only 54/385 (14.0\%) in the group that did not receive PMRT. 

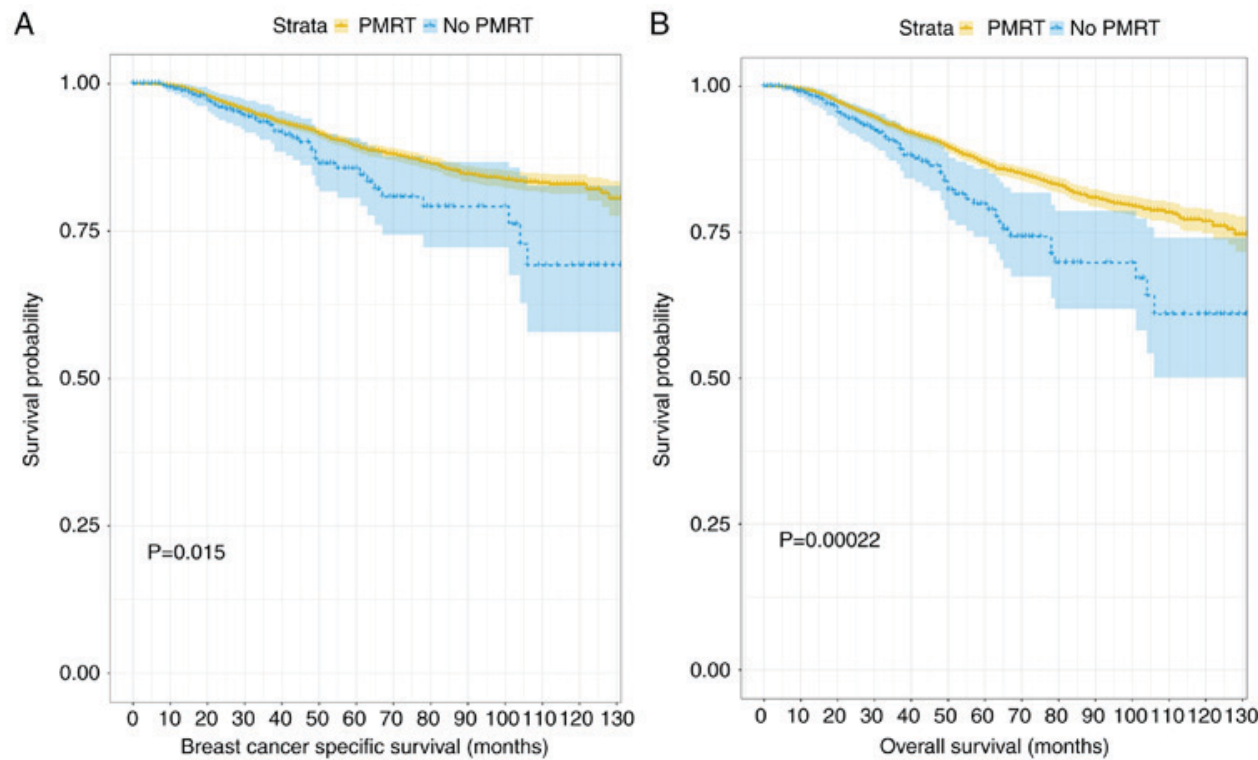

Figure 1. Kaplan-Meier curves for outcomes among patients stratified by the treatment status of radiation prior to matching. (A) Kaplan-Meier survival curve of breast cancer specific survival between the PMRT group and no PMRT group before matching. (B) Kaplan-Meier survival curve of overall survival between the PMRT group and no PMRT group before matching. PMRT, post-mastectomy radiotherapy.
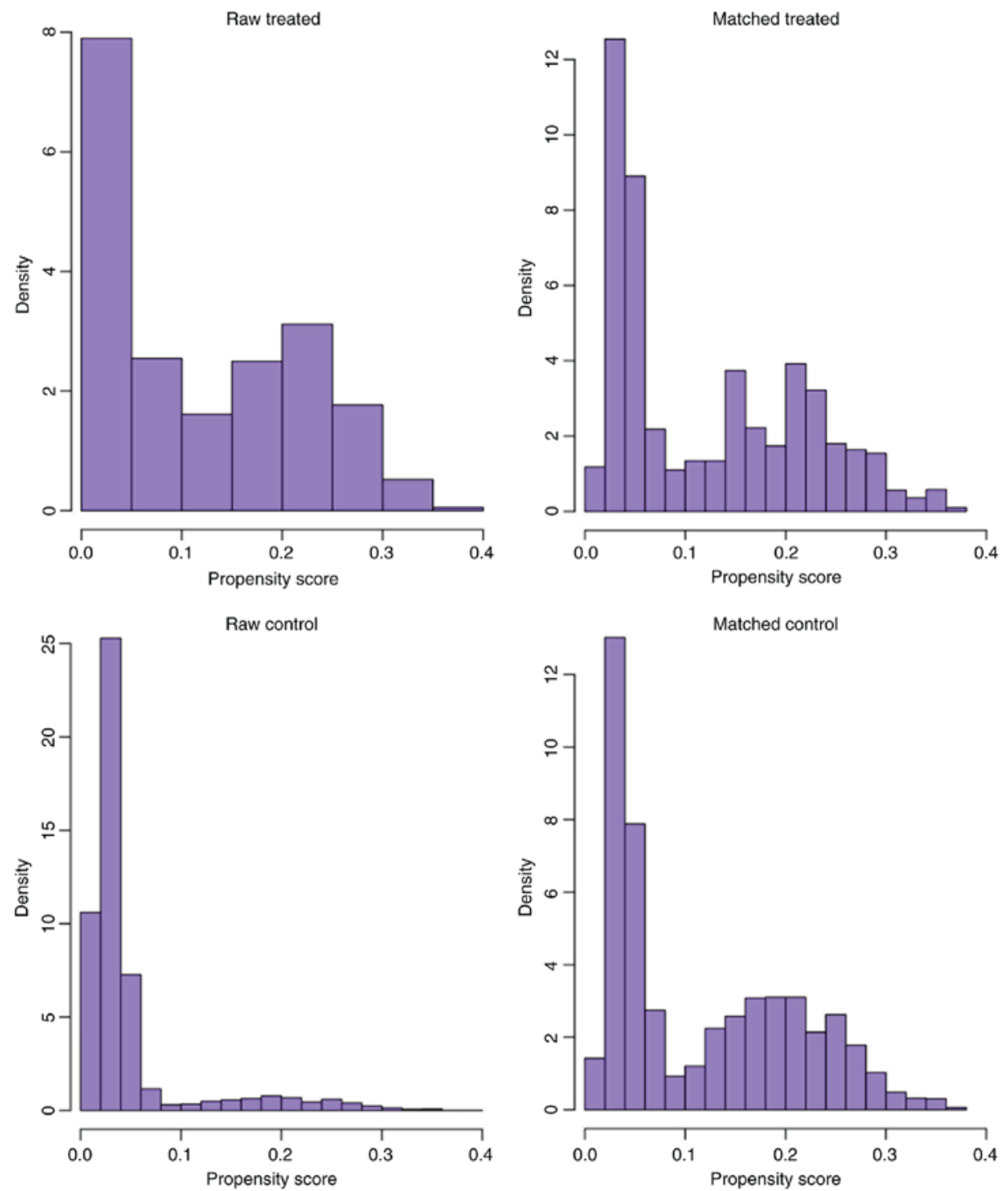

Figure 2. Overlay of histogram distributions prior to and following match. 


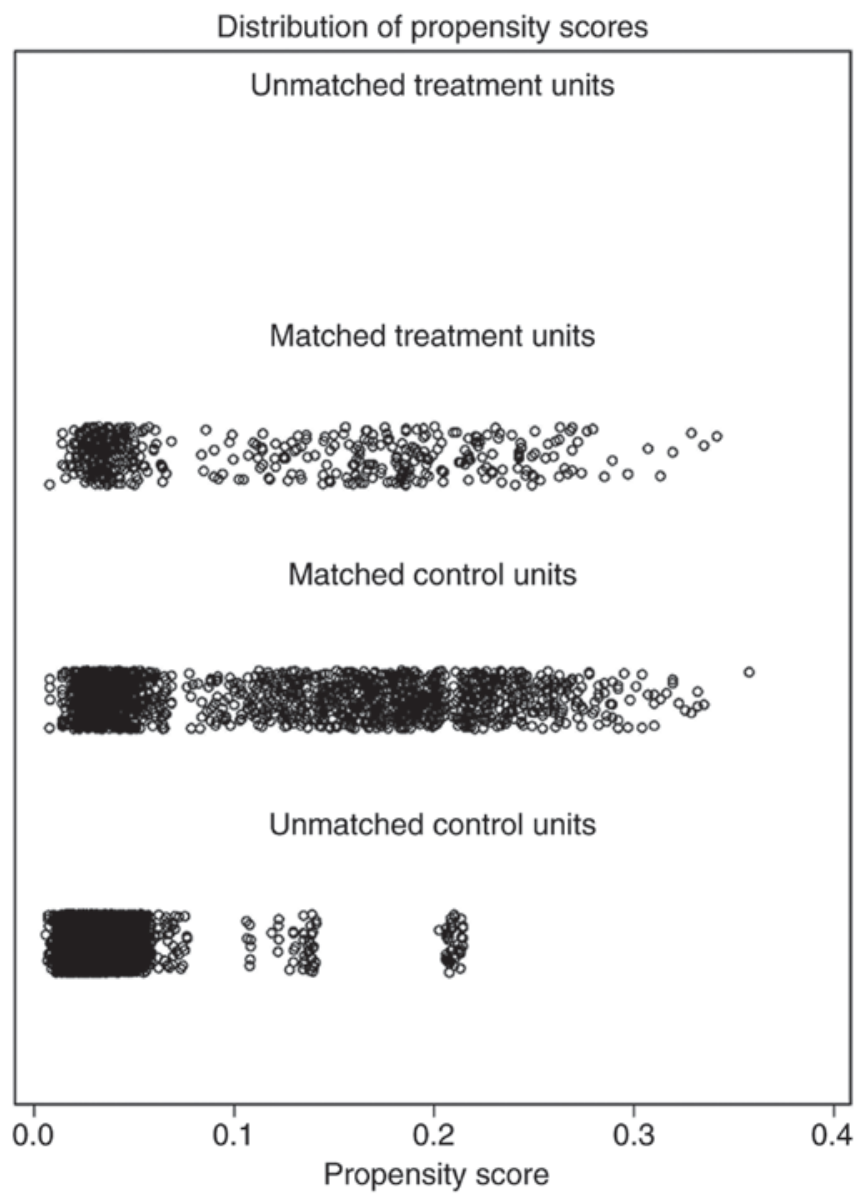

Figure 3. Propensity scores prior to and following propensity score matching.

Fig. 4 depicts the generated Kaplan-Meier curves for outcomes among patients according to their treatment status (PMRT or no PMRT) following propensity score matching. There were statistically significant differences in the BCSS and OS probabilities according to radiotherapy treatment status, with a trend towards improved outcomes in patients who received PMRT. When that data were divided into two further groups according to age ( $\leq 40$ and $>40$ years old) and the differences between BCSS and OS probabilities were compared, the results demonstrated that PMRT had no significant effect on the outcome in the younger age group, despite the trend towards separation in the Kaplan-Meier curves displayed $(\mathrm{P}=0.27$ and $\mathrm{P}=0.33$, respectively; Fig. 5). When the data associated with the older group of patients ( $>40$ years old) were analyzed, patients who had received PMRT exhibited an improved prognosis in terms of BCSS and OS scores ( $\mathrm{P}=0.029$ and $\mathrm{P}=0.002$; Fig. 6). The subgroups were further analyzed by age ( $\leq 40$ and $>40$ years old), and the results demonstrated that PMRT was not an independent prognostic factor for young patients, specifically regarding OS or BCSS ( $\mathrm{P}=0.284$ vs. $\mathrm{P}=0.22$, respectively). By contrast, PMRT demonstrated a statistically significant difference in OS and BCSS in the elderly group ( $\mathrm{P}=0.008$ vs. $\mathrm{P}=0.015$, respectively).

\section{Discussion}

Although it has been reported in the literature that postoperative radiotherapy may be beneficial for improving the prognosis of early-stage breast cancer, the majority of the studies that have been conducted to date have been limited to a single center and employed a small sample size $(22,23)$. To the best of our knowledge, there are only a large number of retrospective studies conducted by Huo et al (24) in 2015. It focuses on the association between the number of early-stage breast cancer LNs and postoperative radiotherapy. The present study analyzed the impact of PMRT on the tumor-specific survival rates of patients with early-stage breast cancer within a large sample drawn from multiple centers, with the aim of improving the evidence for the use of adjunct therapy for early-stage breast cancer following MAS.

The data analysis generated evidence demonstrating that PMRT was associated with improved BCSS and OS probabilities, compared with not receiving PMRT, and the direction of the association was not altered by adjustment of confounding factors. Kindts et al (25) reported a similar observation in a large respective study involving 439 patients with early-stage triple negative breast cancer. Furthermore, the results are consistent with those of another retrospective study by Kim et al (26). The collective evidence, indicating a survival benefit of PMRT, suggests that postoperative radiotherapy may benefit the prognosis of patients with early-stage breast cancer.

A notable observation of the present study was that the tumor location did not significantly impact BCSS probabilities. Conversely, previous studies reported that patients with tumors in the upper-outer quadrant of both sides of the breast, as well as the right central portion, have an improved prognosis, compared with patients with tumors at other locations $(27,28)$. Notably, the assignment of patients to groups in the two studies employed slightly different protocols, which may have resulted in a significant number of false positives being assigned in the effect of primary site on BCSS by Yang et al (27). The other possible cause of the differences in these data is the different statistical tests used to analyze the data.

The present study identified age, race, number of positive nodes and T stage as predictive of BCSS probability following MAS. This observation is consistent with a population-based study, which determined that BCSS score is dependent on the histological grade of the tumor, Tumor-Node-Metastasis stage, LNs, positive hormone receptor status and patient race $(29,30)$. In another study involving a multivariate analysis, Hanrahan et al (31) reported similar significant predictive factors of BCSS. Furthermore, the data of the present study are also supported by a systematic review that summarized the prognostic factors associated with breast cancer (32).

Previous retrospective studies reported inconsistent data with regard to the effect of PMRT on the BCSS and OS probabilities of young patients with breast cancer $(33,34)$. Although the present results indicated that PMRT can extend BCSS and OS in young patients with breast can0cer, the area remains controversial, and whether there are clear benefits of PMRT in younger patients with breast cancer remains to be conclusively demonstrated. Furthermore, when the older patients ( $>40$ years old) were analyzed, an improved prognosis with respect to BCSS and OS by PMRT was indicated in the present study. This observation is consistent with those of Yancik et al (35), who conducted a retrospective study of the effect of various factors, including age of patient outcomes. 
A

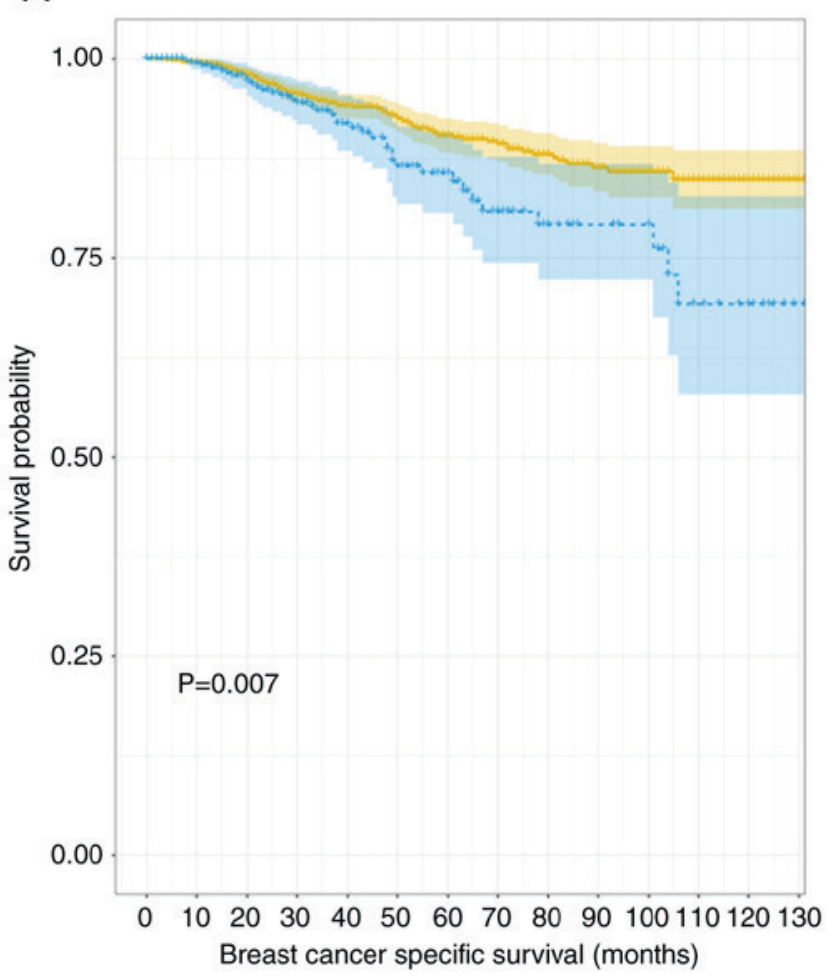

B

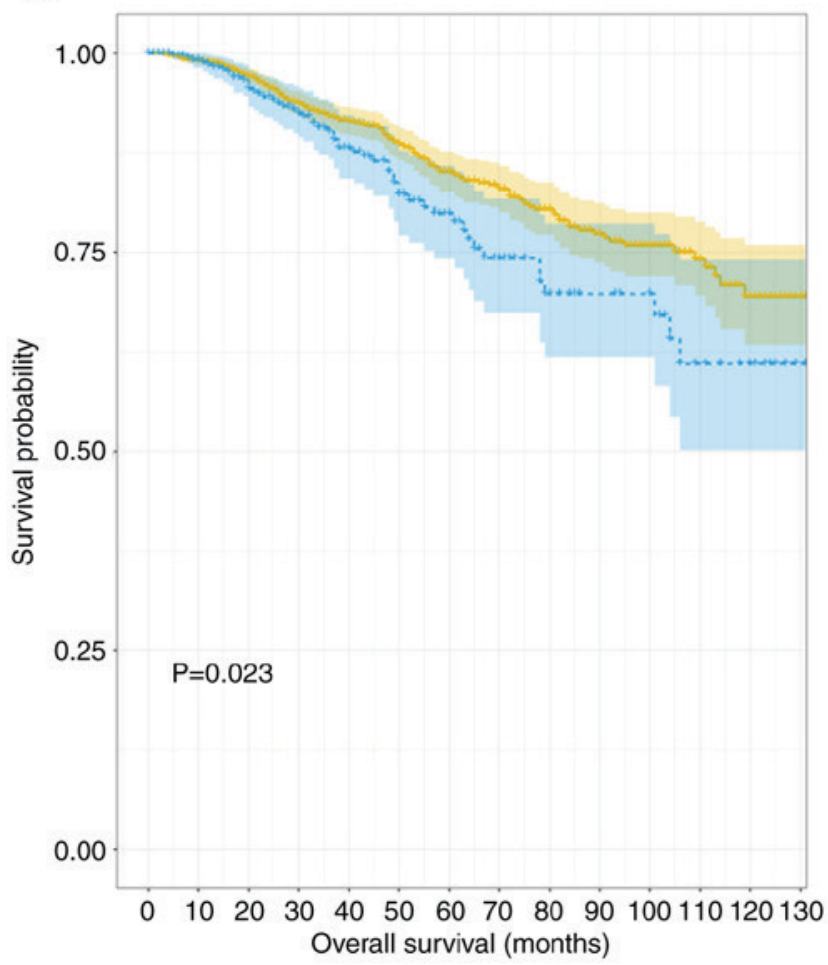

Figure 4. Kaplan-Meier curves for outcomes among patients stratified by the treatment status of radiation following matching. (A) Kaplan-Meier survival curve of breast cancer specific survival between the PMRT group and no PMRT group after matching (B) Kaplan-Meier survival curve of overall survival between the PMRT group and no PMRT group after matching. PMRT, post-mastectomy radiotherapy.

A

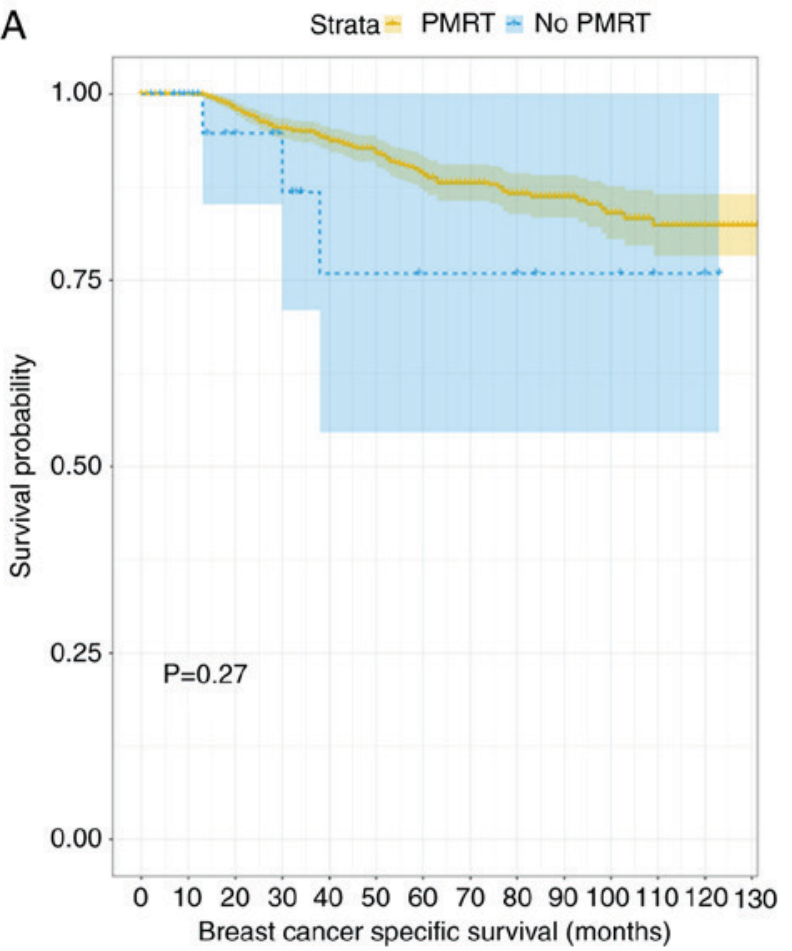

B

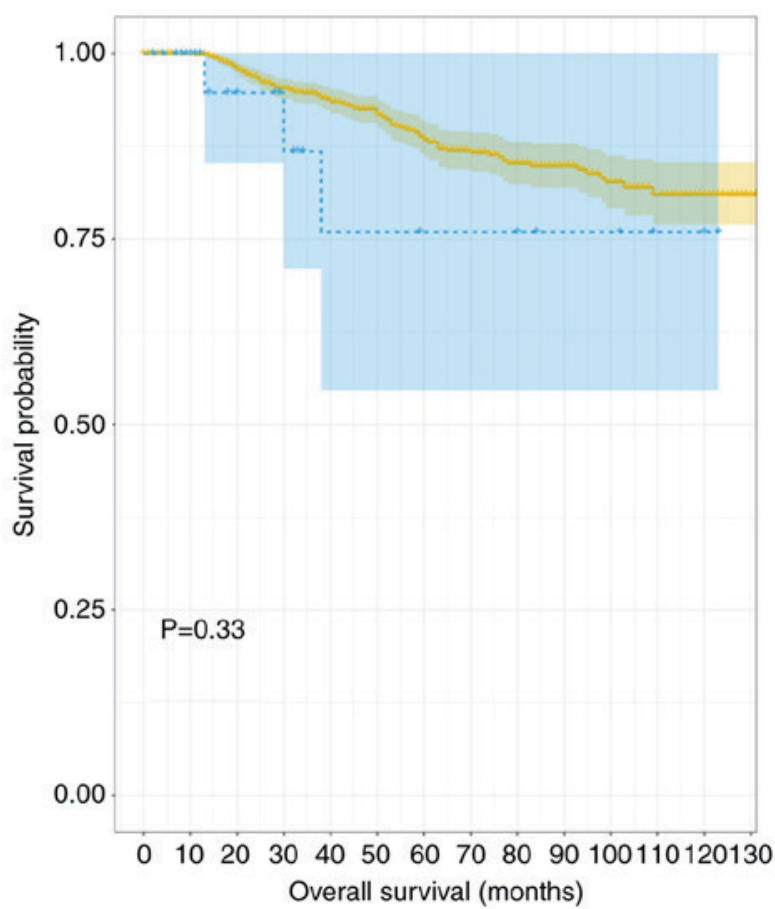

Figure 5. Kaplan-Meier curves for breast cancer-specific survival and overall survival among young group ( $\leq 40$ years). (A) Kaplan-Meier survival curve of breast cancer specific survival in the young group. (B) Kaplan-Meier survival curve of overall survival in the young group. PMRT, post-mastectomy radiotherapy.

The present study has certain limitations. Firstly, the propensity score matching analysis reduced the sample size and as a result the statistical estimations may not be as precise (36). Secondly, although in theory propensity score 
A

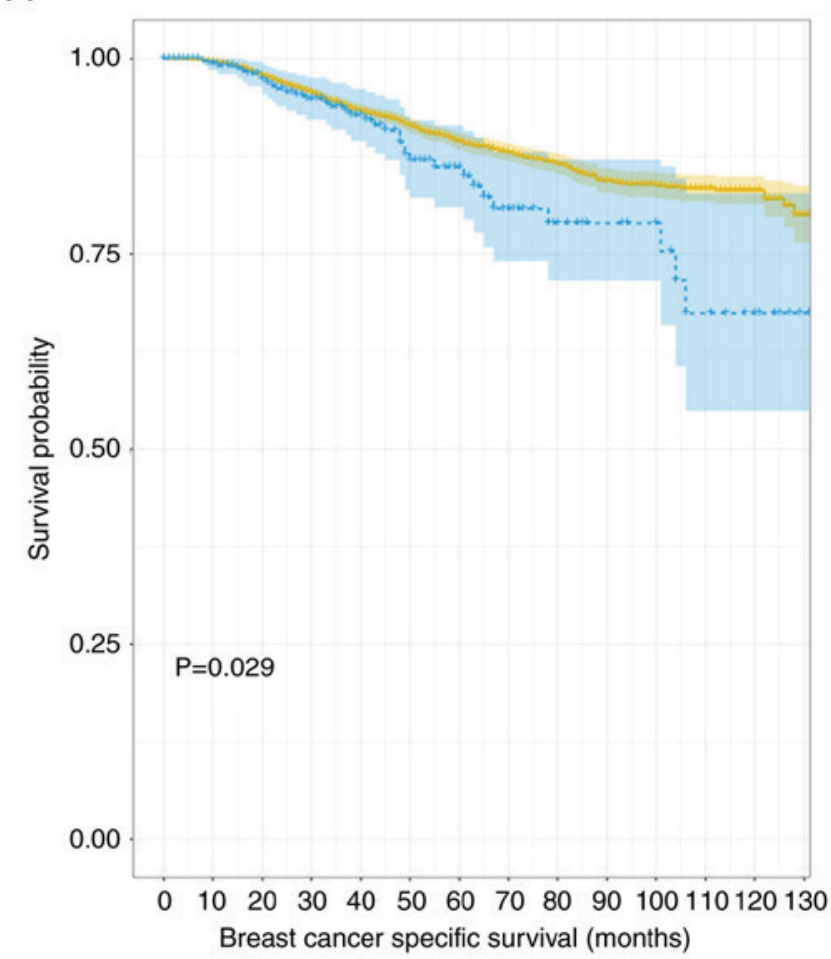

B

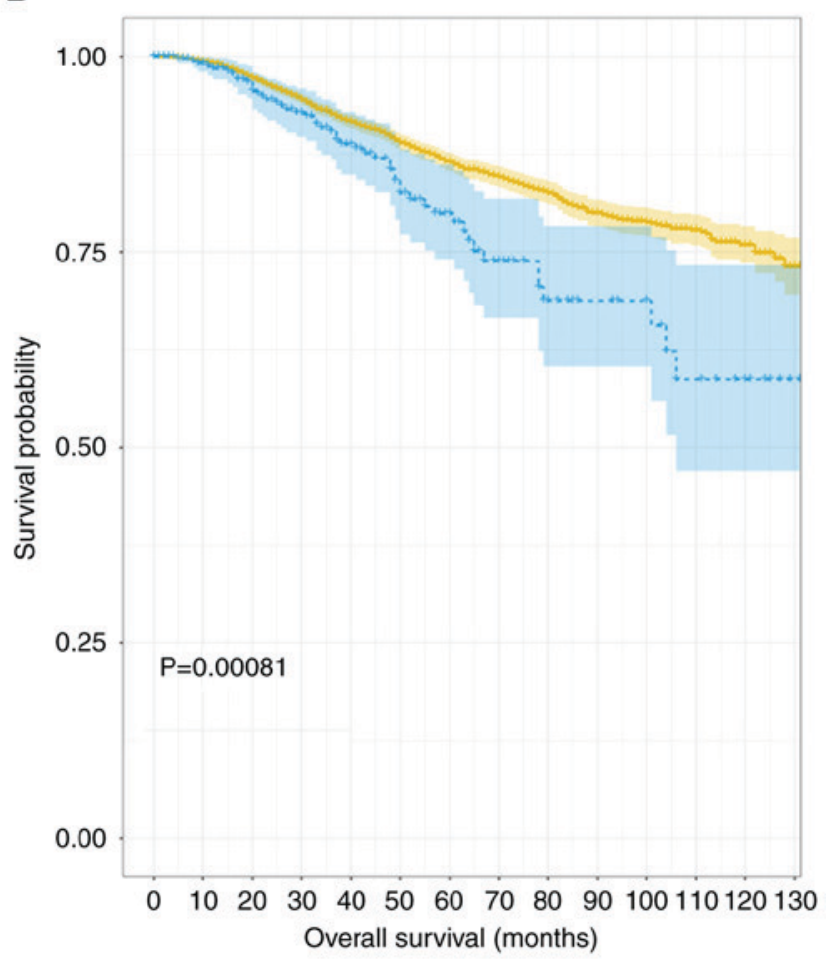

Figure 6. Kaplan-Meier curves for breast cancer-specific survival and overall survival among old group (>40 years). PMRT, post-mastectomy radiotherapy. (A) Kaplan-Meier survival curve of breast cancer specific survival in the older patient group. (B) Kaplan-Meier survival curve of overall survival in the older group of patients.

matching balances covariates between treatment groups, this is not a certainty, particularly with regard to unmeasured variables, including the subtype of breast cancer or type of chemotherapy for breast cancer. These and other potential confounding factors may also impact the predicted outcomes in patients with breast cancer (37). Finally, previous studies analyzed additional outcome measures. These studies demonstrated that PMRT may significantly reduce the local tumor recurrence (LRR) rate in patients with early-stage breast cancer. Notably, relevant data was not available in the context of the present study to analyze for an association between PMRT and LRR.

In conclusion, the present study demonstrated that PMRT may have a positive impact on the clinical outcomes of patients with breast cancer whose cancer is at an early stage. Notably, BCSS and OS probabilities were significantly improved among patients who had received PMRT. These observations indicated that patients with early-stage (T1-2) breast cancer and those with one to three positive axillary LNs should be considered for PMRT prior to performing surgery.

\section{Acknowledgements}

Not applicable.

\section{Funding}

The study was supported by the National Natural Science Foundation of China (grant no.81360418) and the Natural Science Foundation of Guang Xi (grant no. 2014GXNSFCA118011).

\section{Availability of data and materials}

The datasets used and/or analyzed during the current study are available from the corresponding author on reasonable request.

\section{Authors' contributions}

WP designed the experiment, provided financial support, revised the manuscript and gave final approval of the version to be published. WS and YL performed the statistical analysis and wrote the paper. DZ and $\mathrm{HH}$ made substantive contibutions to the work, including data collecting and manuscript revising.

\section{Ethics approval and consent to participate}

Not applicable.

\section{Patient consent for publication}

Not applicable.

\section{Competing interests}

The authors declare that they have no competing interests.

\section{References}

1. Anastasiadi Z, Lianos GD, Ignatiadou E, Harissis HV and Mitsis M: Breast cancer in young women: An overview. Updates Surg 69: 313-317, 2017. 
2. Dimitrova N, Znaor A, Agius D, Eser S, Sekerija M, Ryzhov A Primic-Žakelj M and Coebergh JW; SEE+ Working Group: Breast cancer in South-Eastern European countries since 2000: Rising incidence and decreasing mortality at young and middle ages. Eur J Cancer 83: 43-55, 2017.

3. Mogal HD, Clark C, Dodson R, Fino NF and Howard-Mcnatt M: Outcomes after mastectomy and lumpectomy in elderly patients with early-stage breast cancer. Ann Surg Oncol 24: 100-107, 2017.

4. Mahmood U, Hanlon AL, Koshy M, Buras R, Chumsri S, Tkaczuk KH, Cheston SB, Regine WF and Feigenberg SJ Increasing national mastectomy rates for the treatment of early stage breast cancer. Ann Surg Oncol 20: 1436-1443, 2013.

5. Veronesi U, Cascinelli N, Mariani L, Greco M, Saccozzi R, Luini A, Aguilar M and Marubini E: Twenty-year follow-up of a randomized study comparing breast-conserving surgery with radical mastectomy for early breast cancer. N Engl J Med 347: 1227-1232, 2002.

6. Ursino S, Fiorica F, Mazzotti V, Delishaj D, Cristaudo A, Spagnesi S, Laliscia C, Pasqualetti F, Fontana A, Ghilli M, et al The role of clinicopathologic and molecular prognostic factors in the post-mastectomy radiotherapy (PMRT): A retrospective analysis of 912 patients. Eur Rev Med Pharmacol Sci 21: 2157-2166, 2017.

7. Zhang X, Sun Y and Zhang L: Influence of postoperative radiotherapy on the prognosis of patients with breast cancer in T_1- T_2. J Modern Oncol, 2016.

8. Li XP, Bai GP and Wang ZG: Influence factors on the quality of life for postoperative radiotherapy patients with breast cancer. Anhui Medical \& Pharmaceutical Journal, 2017.

9. Bloomfield DJ; Core Group facilitated by The Royal College of Radiologists: Development of postoperative radiotherapy for breast cancer: UK consensus statements-a model of patient, clinical and commissioner engagement? Clin Oncol (R Coll Radiol) 29: 639-641, 2017.

10. Wang S, He M, Jin Y, Wyss R, Shin H, Ma Y, Keeton S, Fireman B, Karami S, Major JM, et al: A review of the performance of different methods for propensity score matched subgroup analyses and a summary of their application in peer-reviewed research studies. Pharmacoepidemiol Drug Saf 26: 1507-1512, 2017.

11. Francis SR, Frandsen J, Kokeny K, Gaffney D and Poppe M: (P011) postmastectomy radiotherapy for T3N0 breast cancers: A national cancer database analysis. Int J Radiation Oncol Biol Phy 98: E18, 2017.

12. Park HJ, Shin KH, Kim JH, Ahn SD, Kim SS, Kim YB, Park W, Kim YJ, Shin HS, Kim JH, et al: Possible benefits from post-mastectomy radiotherapy in node-negative breast cancer patients: A multicenter analysis in Korea (KROG 14-22). Oncotarget 8: 59800-59809,2017.

13. Forissier V, Tallet A, Cohen M, Classe JM, Reyal F, Chopin N, Mazouni C, Gimbergues P, Daraï E, Colombo PE, et al: Is post-mastectomy radiation therapy contributive in $\mathrm{pN} 0-1 \mathrm{mi}$ breast cancer patients? Results of a French multi-centric cohort. Eur J Cancer 87: 47-57, 2017.

14. Belkacémi Y, Comet B, Hannoun-Levi JM, Villette S, Marsiglia H, Leblanc-Onfroy M, Delalande B, Azria D and Dubois JB: Accelerated partial breast irradiation: A concept to individualize treatment in breast cancer. Cancer Radiother 11: 287-295, 2007 (In French).

15. Zhou SY, Xue Q, Ying JM, Hu XS, Yang JL, Lin H and Shi YK: The clinical and pathological features, biomarker characteristics and prognosis analysis of lung adenosquamous carcinoma. Zhonghua Zhong Liu Za Zhi 41: 50-55, 2019 (In Chinese).

16. Thoemmes FJ and Kim ES: A systematic review of propensity score methods in the social sciences. Multivariate Behav Res 46: 90-118, 2011

17. Li FQ and Hui MA: Sensitivity analysis on the propensity score matching method. Statistics and Information Forum, 2009.

18. Fauser D and Bethge M: Propensity score methods to estimate treatment effects: An opportunity for rehabilitation service research. Rehabilitation (Stuttg) 58: 50-58, 2019 (In German).

19. Cuccurullo V and Mansi L: AJCC cancer staging handbook: From the AJCC cancer staging manual (7th edition). Eur J Nucl Med Mol Imag 38: 408-408, 2011.

20. Pike MC and Morrow RH: Statistical analysis of patient-control studies in epidemiology. Factor under investigation an all-or-none variable. Br J Prev Soc Med 24: 42-44, 1970.
21. Pike MC, Casagrande J and Smith PG: Statistical analysis of individually matched case-control studies in epidemiology: Factor under study a discrete variable taking multiple values. Br J Prev Soc Med 29: 196-201, 1975.

22. Kim CS and Algan O: Radiation Therapy, Breast Cancer Early Stage. Stat Pearls Stat Pearls Publishing Stat Pearls Publishing, LLC, Treasure Island (FL), 2017.

23. Pérez M, Schootman M, Hall LE and Jeffe DB: Accelerated partial breast irradiation compared with whole breast radiation therapy: A breast cancer cohort study measuring change in radiation side-effects severity and quality of life. Breast Cancer Res Treat 162: 329-342, 2017.

24. Huo D, Hou N, Jaskowiak N, Winchester DJ, Winchester DP and Yao K: Use of postmastectomy radiotherapy and survival rates for breast cancer patients with T1-T2 and one to three positive lymph nodes. Ann Surg Oncol 22: 4295-4304, 2015.

25. Kindts I, Buelens P, Laenen A, Van Limbergen E, Janssen H, Wildiers $\mathrm{H}$ and Weltens $\mathrm{C}$ : Omitting radiation therapy in women with triple-negative breast cancer leads to worse breast cancer-specific survival. Breast 32: 18-25, 2017.

26. Kim B, Kwon J and Kim K: Evaluation of the benefit of radiotherapy in patients with occult breast cancer: A population-based analysis of the SEER database. Cancer Res Treat 50: 551-561, 2018.

27. Yang J, Tang S, Zhou Y, Qiu J,Zhang J, Zhu S and Lv Q: Prognostic implication of the primary tumor location in early-stage breast cancer: Focus on lower inner zone. Breast Cancer 25: 100-107, 2018.

28. Hwang KT, Kim J, Kim EK, Jung SH, Sohn G, Kim SI, Jeong J, Lee HJ, Park JH and Oh S; Korean Breast Cancer Society: Poor prognosis of lower inner quadrant in lymph node-negative breast cancer patients who received no chemotherapy: A study based on nationwide korean breast cancer registry database. Clin Breast Cancer 17: e169-e184, 2017.

29. Dawood S, Lei X, Dent R, Gupta S, Sirohi B, Cortes J, Cristofanilli M, Buchholz T and Gonzalez-Angulo AM: Survival of women with inflammatory breast cancer: A large population-based study. Ann Oncol 25: 1143-1151, 2014.

30. DeBono NL, Robinson WR, Lund JL, Tse CK, Moorman PG, Olshan AF and Troester MA: Race, menopausal hormone therapy, and invasive breast cancer in the carolina breast cancer study. J Womens Health (Larchmt) 27: 377-386, 2018.

31. Hanrahan EO, Gonzalez-Angulo AM, Giordano SH, Rouzier R, Broglio KR, Hortobagyi GN and Valero V: Overall survival and cause-specific mortality of patients with stage T1a,bNOM0 breast carcinoma. J Clin Oncol 25: 4952-4960, 2007.

32. Howell A, Anderson AS, Clarke RB, Duffy SW, Evans DG, Garcia-Closas M, Gescher AJ, Key TJ, Saxton JM and Harvie MN: Risk determination and prevention of breast cancer. Breast Cancer Res 16: 446, 2014.

33. Park HL, Chang J, Lal G, Lal K, Ziogas A and Anton-Culver H: Trends in treatment patterns and clinical outcomes in young women diagnosed with ductal carcinoma in situ. Clin Breast Cancer 18: e179-e185, 2018

34. Chow E: Radiation treatment for breast cancer. Recent advances. Can Fam Physician 48: 1065-1069, 2002.

35. Yancik R, Wesley MN, Ries LA, Havlik RJ, Edwards BK and Yates JW: Effect of age and comorbidity in postmenopausal breast cancer patients aged 55 years and older. JAMA 285: 885-892, 2001.

36. von Elm E, Altman DG, Egger M, Pocock SJ, Gøtzsche PC and Vandenbroucke JP; STROBE Initiative: The strengthening the reporting of observational studies in epidemiology (STROBE) statement: Guidelines for reporting observational studies. Prev Med 45: 247-251, 2007.

37. Seki T, Jinno H, Okabayashi K, Murata T, Matsumoto A, Takahashi M, Hayashida T and Kitagawa Y: Comparison of oncological safety between nipple sparing mastectomy and total mastectomy using propensity score matching. Ann R Coll Surg Eng1 97: 291-297, 2015.

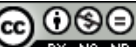

This work is licensed under a Creative Commons Attribution-NonCommercial-NoDerivatives 4.0 International (CC BY-NC-ND 4.0) License. 